\title{
Wireless Transmission of Big Data: A Transmission Time Analysis over Fading Channel
}

\author{
Wen-Jing Wang, Student Member, IEEE Hong-Chuan Yang, Senior Member, IEEE, Mohamed-Slim Alouini, \\ Fellow, IEEE
}

\begin{abstract}
In this paper, we investigate the transmission time of a large amount of data over fading wireless channel with adaptive modulation and coding (AMC). Unlike traditional transmission systems, where the transmission time of a fixed amount of data is typically regarded as a constant, the transmission time with AMC becomes a random variable, as the transmission rate varies with the fading channel condition. To facilitate the design and optimization of wireless transmission schemes for big data applications, we present an analytical framework to determine statistical characterizations for the transmission time of big data with AMC. In particular, we derive the exact statistics of transmission time over block fading channels. The probability mass function (PMF) and cumulative distribution function (CDF) of transmission time are obtained for both slow and fast fading scenarios. We further extend our analysis to Markov channel, where transmission time becomes the sum of a sequence of exponentially distributed time slots. Analytical expression for the probability density function (PDF) of transmission time is derived for both fast fading and slow fading scenarios. These analytical results are essential to the optimal design and performance analysis of future wireless transmission systems for big data applications.
\end{abstract}

Index Terms-Transmission time, adaptive modulation and coding, block fading, Markov channel, distribution functions.

\section{INTRODUCTION}

$\mathbf{W}$ $\mathrm{E}$ are entering a big data era. Data are generated and collected at an accelerating rate. The timely processing, delivery, and analysis of these data can bring huge social and economical benefit [1]-[3]. With the intense of recent and ongoing deployment of wireless communication systems, much of the big data will be transmitted wirelessly. In fact, smart mobile devices contribute significantly to the generation of big data [4]. Over the past several years, mobile data traffic has been experiencing a compound annual growth rate of over $40 \%$. This growth rate is expected to accelerate in the coming years as the result of increasing popularity of mobile broadband applications. The supporting of big data transmission presents several technical challenges to wireless systems design, including spectrum efficiency enhancement of radio access network (RAN), capacity provision of fronthaul/backhaul links, and network architecture improvement

Manuscript received December 1, 2012; revised August 26, 2015.

W.-J. Wang is with the Department of Electrical and Computer Engineering, University of Victoria, Victoria, BC V8W 2Y2, Canada (e-mail: wjing@uvic,ca)

H.-C. Yang is with the Department of Electrical and Computer Engineering, University of Victoria, Victoria, BC V8W 2Y2, Canada (e-mail: hy@uvic,ca)

M.-S. Alouini is with the Computer, Electrical and Mathematical Science and Engineering Division, King Abdullah University of Science and Technology, Thuwal 23955, Saudi Arabia (e-mail: slim.alouini@kaust.edu.sa). for traffic scalability. In this work, we investigate the efficient and reliable transmission of big data over fading wireless channels. In particular, we study the wireless transmission of big data from a transmission time perspective.

Transmission time refers to the time duration required to transmit a certain amount of data and has many important applications in wireless communication system analysis and design. Specifically, transmission time is closely related to the service time in the queuing setup, which is essential to the delay and throughput analysis of various wireless transmission strategies [5]-[7]. Transmission time was applied to investigate the extended delivery time of secondary transmission with interweave cognitive radio implementation [8], [9]. Transmission time also serves as a characterization of channel occupancy. As such, the distribution of transmission time directly affects the collision probability of random access protocols over wireless channels [10]. Furthermore, as the energy consumption of transmitter can be calculated as the product of transmission time and transmit power, transmission time analysis is instructive in designing energy-efficient wireless communication systems [11]-[13]. To effectively support big data transmission, the transmission strategies, access protocols, and scheduling policies of future wireless systems need to be adapted or redesigned [14]. Transmission time characterization for big data traffics over fading wireless channel is of critical importance in this process.

Previous works typically assume a constant transmission time for a fixed amount of data [7], [8], [10], [15]. The transmission time over a point-to-point link is typically calculated as the ratio of the amount of data over the transmission rate when the transmission rate is constant. The minimum transmission time with constant-rate transmission can be estimated as $H_{t} /\left(B \log _{2}(1+\gamma)\right)$, where $H_{t}$ is the data size, $B$ is the channel bandwidth and $\gamma$ is the received signal to noise ratio (SNR). Meanwhile, constant-rate transmission becomes inefficient and unreliable when operating over fading wireless channels. Adaptive transmission is an attractive technology to improve the transmission efficiency over wireless channels with guaranteed reliability [16]-[18]. With rate adaptive transmission, the transceiver can adjust the transmission rate with the prevailing channel condition, exploring favorable channel condition with higher rate transmission and maintaining reliability with lower rate for poor channel quality. Assuming continuous rate adaptation, authors of [19] derive the distribution function of transmission time over fading channels through a transformation of random variable. [15] analyzes packet transmission time of cognitive radio network with 
power adaptation considering primary interference constraint. These results, however, are only applicable to the case where data transmission completes with one channel coherence time. On the other hand, the transmission of big data will usually involve multiple channel coherence time, even for slow fading environment. The transformation of random variable approach adopted in [15], [19] can not apply to the transmission time analysis for big data over wireless channels. To the best knowledge of the authors, the transmission time of a large amount of data over fading channels with rate adaptation has been barely investigated in the literature.

In this paper, we present an analytical framework to investigate the transmission time of big data over fading wireless channels. We consider a generic point-to-point transmission scenario where adaptive modulation and coding (AMC) is adopted. AMC is a practical rate adaptive transmission scheme [20]-[23] that can approach the performance of continuous rate adaptation. With AMC, the modulation/coding scheme is adjusted with instantaneous channel condition, while maintaining an acceptable bit error rate (BER) performance. As such, AMC is suitable candidate for the efficient and reliable transmission of big data over fading wireless channels. Meanwhile, the transmission time becomes random and depends on the channel realization when AMC is applied. To facilitate the delay/throughput analysis and energy efficiency optimization of big data transmission over wireless environment, we develop the complete statistical characterization of the transmission time of big data over fading channels in this work. We first consider block fading channels and derive the exact distribution functions of transmission time for both slow and fast fading scenarios. Then, we generalize the analysis to Markov channel scenario, for which the exact probability density function (PDF) and approximate PDF of the transmission time is derived for slow fading and fast fading scenarios, respectively.

The contributions of this work can be summarized as follow:

1) We propose a general analytical framework for the transmission time of big data with AMC over fading wireless channels. Most previous works assumed constant rate transmission. We generalize the analysis by allowing variable transmission rate, which is especially applicable to big data transmission.

2) The exact distribution functions of transmission time over block fading channels are derived. To reduce the computation complexity, we also obtain an approximate probability mass function (PMF) of the transmission time in fast fading scenario.

3) For Markov channels, the exact PDF of transmission time is derived assuming that data transmission completes within a few channel coherence time, which is applicable for big data transmission over slow fading environment. For fast fading scenario, the approximate PDF of transmission time is also obtained using the central limit theorem.

4) Selected numerical examples are presented and discussed to illustrate the mathematical formulations. We show that the transmission time of big data with AMC may vary dramatically with the prevailing fading channel

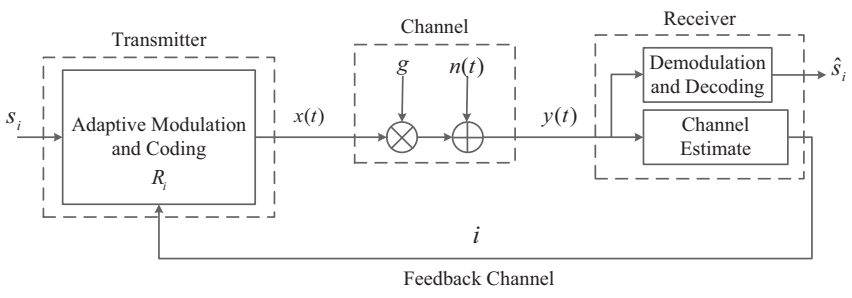

Fig. 1. Illustration of adaptive modulation and coding system over fading channels.

condition. These analytical results will greatly facilitate further improvement of wireless transmission schemes for big data applications.

The rest of this paper is arranged as follows. Section II introduces system and channel model for big data transmission with AMC. In Section III, we study transmission time over block fading channels. In Section IV, we extend the transmission time analysis to Markov channels. Select numerical results are presented and discussed in Section V. The conclusion is presented in Section VI.

\section{System AND ChANNEl Model}

We consider a generic digital transmission system for big data applications operating over flat fading wireless channel, as shown in Fig. 1. Specifically, the information bits $s_{i}$ are coded and modulated to generate the transmitted signal $x(t)$. The wireless channel introduces flat fading channel gain $g$ and additive white Gaussian noise (AWGN) $n(t)$, which leads to the received signal $y(t)=g x(t)+n(t)$. The receiver tries to recover the information bits from the received signal $y(t)$. We assume that the receiver performs coherent detection on the received signal with perfect channel phase estimate. As such, the instantaneous channel quality is characterized by the instantaneous received SNR defined as $\gamma=\frac{|g|^{2} E_{s}}{N_{0}}$, where $E_{s}$ denotes the symbol energy and $N_{0}$ is the power spectral density of the additive noise.

The transmission system adopts constant-power discreterate adaptation with AMC. In particular, the data rate is adaptively adjusted based on the fading channel quality by using different modulation-coding schemes, while the transmission power remains constant. More specifically, the value range of received SNR is divided into $N$ regions, $A_{i}=\left[\gamma_{i-1}, \gamma_{i}\right), i=$ $1,2, \ldots, N$, with $\gamma_{0}=0$ and $\gamma_{N}=\infty$. When the received SNR $\gamma$ falls in region $A_{i}$, the system will use a modulationcoding scheme with data rate $R_{i}$ bits/symbol, $i=1,2, \cdots N$. $R_{1}$ could be zero to account for the outage state. The boundary SNRs $\gamma_{i}, i=1,2, \cdots, N-1$, are typically determined such that the instantaneous BER for selected modulation-coding scheme is below a target BER value, denoted by BER $\mathrm{tar}_{\operatorname{tar}}$ [24], [25]. The probability of using transmission rate $R_{i}$, denoted by $\pi_{i}$, can then be calculated as the probability that $\gamma$ falls into region $A_{i}$, i.e.

$$
\pi_{i}=\operatorname{Pr}\left[\gamma \in A_{i}\right]=\int_{\gamma_{i-1}}^{\gamma_{i}} f_{\gamma}(\gamma) d \gamma
$$




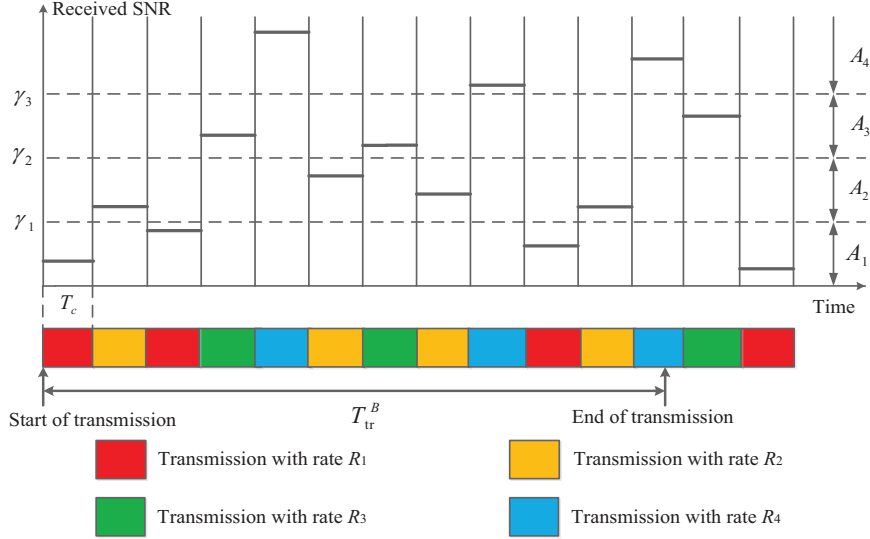

Fig. 2. Illustration of transmission time with four-state AMC over block fading.

where $f_{\gamma}(\cdot)$ is the PDF of the received SNR, depending on the chosen fading channel model.

To implement AMC, the receiver needs to estimate the received SNR and determine which SNR region it falls into. The receiver then feeds back the index of the chosen modulationcoding pair to the transmitter via an error-free feedback channel. After that, the transmitter and the receiver communicate using the chosen modulation-coding scheme. The modulation-coding scheme selection should be periodically updated according to the prevailing channel quality, usually once every channel coherence time $T_{c}$. As such, when a large amount of data is transmitted with AMC, the transmission rate may vary during the transmission. The total transmission time will involve multiple channel coherence time. In the following sections, we derive the distribution function of the transmission time for block fading and Markov fading channels.

\section{TRANSMISSION TIME ANALYSIS FOR BLOCK FADING}

In this section, we consider block fading channel, where the channel gain remains constant for one channel coherence time $T_{c}$, and varies independently from one $T_{c}$ to another. As such, the transmission time for block fading, denoted by $T_{\mathrm{tr}}^{B}$, consists a sequence of channel coherence time, as illustrated in Fig. 2. Here, we assume that transmission starts at the beginning of a channel coherence time without loss of generality.

\section{A. Exact expression}

Suppose that data transmission completes over $L$ channel coherence time. The corresponding transmission time, denoted by $T_{\mathrm{tr}, L}^{B}$, is the sum of $L-1$ intact $T_{c}$ 's and one partial $T_{c}$, the length of which depends on the total amount of data $H_{t}$ and the data amount transmitted over first $L-1$ coherence time. Mathematically, the transmission time can be formulated as

$$
T_{\mathrm{tr}, L}^{B}=(L-1) T_{c}+\frac{H_{t}-\mathbb{H}_{L-1}^{B}}{R^{(L)}}, \quad L=1,2, \cdots
$$

where $R^{(L)}$ denotes the data rate used in the $L$ th $T_{c}$ and $\mathbb{H}_{L-1}^{B}$ is the amount of data transmitted over previous $L-1 T_{c}$ 's.
Note that transmission rate over each $T_{c}$ is determined by the corresponding channel realization. Let us assume that, during the first $L-1$ channel coherence time, the received SNR falls into region $A_{i}$ total $n_{i}$ times, $i=1, \cdots, N$, and as such, rate $R_{i}$ is used $n_{i}$ times, where $n_{i}$ 's satisfy that $\sum_{i=1}^{N} n_{i}=L-1$. Accordingly, $\mathbb{H}_{L-1}^{B}$ is given by

$$
\mathbb{H}_{L-1}^{B}=\sum_{i=1}^{N} n_{i} R_{i} T_{c} .
$$

Let vector $\overrightarrow{\mathbf{n}}=\left[n_{1}, n_{2}, \cdots, n_{N}\right]$ represent such channel realization. Applying the block fading assumption, the probability that such channel realization occurs can be calculated as

$$
\operatorname{Pr}[\overrightarrow{\mathbf{n}}]=\left(\begin{array}{c}
L-1 \\
n_{1}, n_{2}, \cdots, n_{N}
\end{array}\right) \prod_{i=1}^{N} \pi_{i}^{n_{i}},
$$

where $\left(\begin{array}{c}L-1 \\ n_{1}, n_{2}, \cdots, n_{N}\end{array}\right)=\frac{(L-1) !}{n_{1} ! n_{2} ! \cdots n_{N} !}$ denotes the multinomial coefficient. Eq. (4) helps us arrive at the following PMF of the amount of data transmitted over first $L-1$ channel coherence time as

$$
\operatorname{Pr}\left[\mathbb{H}_{L-1}^{B}=T_{c} \sum_{i=1}^{N} n_{i} R_{i}\right]=\left(\begin{array}{c}
L-1 \\
n_{1}, n_{2}, \cdots, n_{N}
\end{array}\right) \prod_{i=1}^{N} \pi_{i}^{n_{i}} .
$$

It is important to note that data transmission completes in exact $L$ channel coherence time if and only if the data transmitted over the first $L-1$ coherence time $\mathbb{H}_{L-1}^{B}$ falls into the region $\left(H_{t}-R_{k} T_{c}, H_{t}\right]$, while rate $R_{k}$ is used in the $L$ th $T_{c}$. Noting that rate $R_{k}$ is used in the $L$ th channel coherence time with probability $\pi_{k}$, we can determine the PMF of $T_{\text {tr }}^{B}$, by considering all $L$ values, as

$$
\begin{gathered}
\operatorname{Pr}\left[T_{\mathrm{tr}, L}^{B}=(L-1) T_{c}+\frac{H_{t}-T_{c} \sum_{i=1}^{N} n_{i} R_{i}}{R_{k}}\right]= \\
\left\{\begin{array}{c}
\pi_{k}\left(\begin{array}{c}
L-1 \\
n_{1}, n_{2}, \ldots, n_{N}
\end{array}\right) \prod_{i=1}^{N} \pi_{i}^{n_{i}} \\
0<H_{t}-T_{c} \sum_{i=1}^{N} n_{i} R_{i} \leq R_{k} T_{c} \\
\text { otherwise; } \quad \begin{array}{c}
L=1,2, \cdots
\end{array}
\end{array}\right.
\end{gathered}
$$

For the special case of $L=1$, we have $\mathbb{H}_{L-1}^{B}=0$, which leads to

$$
\operatorname{Pr}\left[T_{\mathrm{tr}, 1}^{B}=\frac{H_{t}}{R_{k}}\right]=\left\{\begin{array}{cc}
\pi_{k}, & 0<H_{t} \leq R_{k} T_{c} \\
0, & \text { otherwise. }
\end{array}\right.
$$

When $L=2$, we have $\mathbb{H}_{L-1}^{B}=R^{(1)} T_{c}$, where $R^{(1)}$ is the rate used in the first $T_{c}$ and equal to $R_{i}$ with probability $\pi_{i}$. The corresponding PMF terms for $L=2$ are given by

$$
\begin{aligned}
\operatorname{Pr}\left[T_{\mathrm{tr}, 2}^{B}=T_{c}+\frac{H_{t}-R_{i} T_{c}}{R_{k}}\right] & = \\
& \left\{\begin{array}{cc}
\pi_{i} \pi_{k}, & 0<H_{t}-R_{i} T_{c} \leq R_{k} T_{c} ; \\
0, & \text { otherwise. }
\end{array}\right.
\end{aligned}
$$

Conditioning on the number of channel coherence time $L$ and the channel realization vector $\overrightarrow{\mathbf{n}}$, the exact CDF of $T_{\mathrm{tr}}^{B}$ 
over block fading channel model, denoted by $F_{T_{\mathrm{tr}}}^{B}(t)$, can be calculated using Eq. (6) as

$$
\begin{aligned}
F_{T_{\mathrm{tr}}}^{B}(t) & =\operatorname{Pr}\left[T_{\mathrm{tr}}^{B} \leq t\right]=\sum_{L} \operatorname{Pr}\left[T_{\mathrm{tr}, L}^{B} \leq t\right] \\
& =\sum_{L} \sum_{\overrightarrow{\mathbf{n}}} \sum_{k=1}^{N} \pi_{k}\left(\begin{array}{c}
L-1 \\
n_{1}, n_{2}, \ldots, n_{N}
\end{array}\right) \prod_{i=1}^{N} \pi_{i}^{n_{i}} \\
& \times \mathcal{I}_{\left(0, R_{k} T_{c}\right]}\left(H_{t}-T_{c} \sum_{i=1}^{N} n_{i} R_{i}\right) \\
& \times \mathcal{U}\left(t-(L-1) T_{c}+\frac{H_{t}-T_{c} \sum_{i=1}^{N} n_{i} R_{i}}{R_{k}}\right),
\end{aligned}
$$

where $\mathcal{U}[\cdot]$ denotes unit step function, $\mathcal{I}_{A}(x)$ is an indicator function, equal to 1 if $x \in A$ and zero otherwise, and $\sum_{\overrightarrow{\mathbf{n}}}$ is carried over all $n_{i} \geq 0, i=1,2, \cdots, N$, subject to $\sum_{i=1}^{N} n_{i}=L-1$. As shown in numerical results, we can achieve sufficient accuracy by summing $L$ over $\left[1,\left\lfloor 3 L_{\text {ave }}^{B}\right\rfloor\right]$, where $L_{\text {ave }}^{B}=H_{t} / T_{c} \sum_{i=1}^{N} R_{i} \pi_{i}$ is the average number of channel coherence time required. The following algorithm illustrates the procedure of calculating the exact $\mathrm{CDF}$ of $T_{\mathrm{tr}}^{B}$.

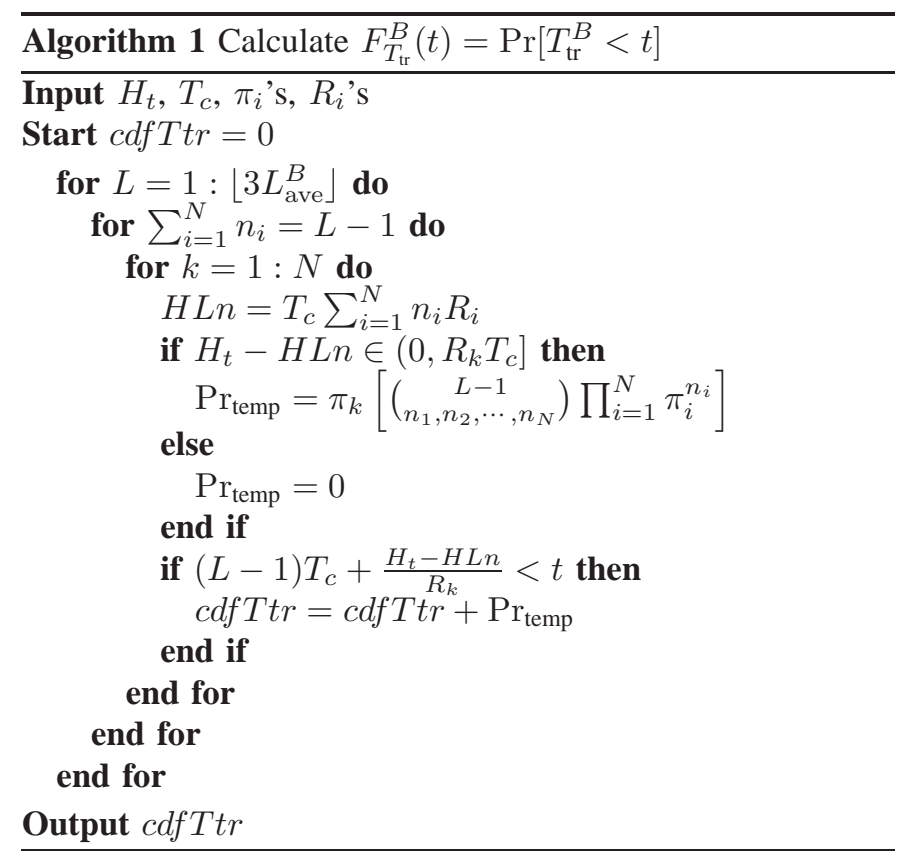

\section{B. Approximate distribution}

When the channel introduces fast fading and $T_{c}$ is very short compared to total transmission time, we can arrive at more convenient expression for PMF of $T_{\text {tr }}^{B}$, by assuming that data transmission always completes in integer number of channel coherence time, (i.e. $T_{\operatorname{tr}, L}^{B}=L T_{c}$,). The probability that transmission completes over $L T_{c}$ 's equals to the joint probability that data transmitted in previous $L-1 T_{c}$ 's is less than $H_{t}$, and data transmitted in $L T_{c}$ 's is greater than $H_{t}$, i.e.

$$
\begin{aligned}
\operatorname{Pr}\left[T_{\mathrm{tr}, L}^{B}\right. & \left.=L T_{c}\right] \\
& =\operatorname{Pr}\left[\mathbb{H}_{L-1}^{B}<H_{t}, \mathbb{H}_{L-1}^{B}+R^{(L)} T_{c} \geq H_{t}\right] .
\end{aligned}
$$

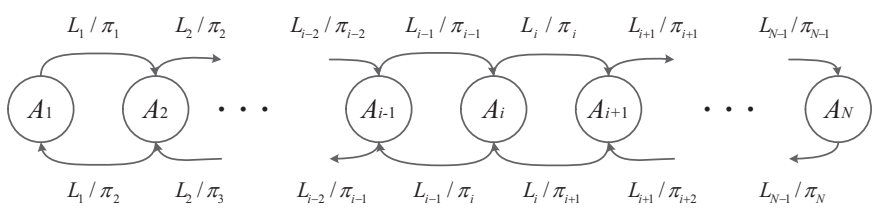

Fig. 3. Illustration of continuous-time finite-state homogeneous Markov model for wireless fading channel.

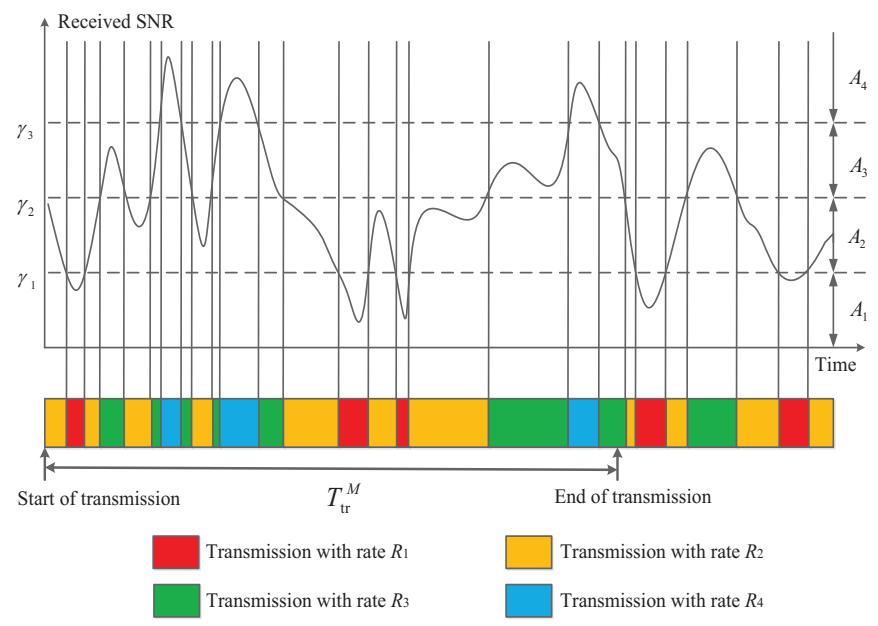

Fig. 4. Illustration of data transmission over Markov channel with AMC.

Conditioning on channel realization over $L$ channel coherence time and applying the result in Eq. (5), we obtain the approximate PMF of $T_{\mathrm{tr}, L}^{B}$ as

$$
\begin{aligned}
& \operatorname{Pr}\left[T_{\mathrm{tr}, L}^{B}=L T_{c}\right] \\
& =\sum_{k=1}^{N} \operatorname{Pr}\left[H_{t}-R_{k} T_{c} \leq \mathbb{H}_{L-1}^{B}<H_{t}\right] \pi_{k} \\
& =\sum_{k=1}^{N}\left(\sum_{\substack{\overrightarrow{\mathbf{n}} \text { s.t. } \\
\mathbb{H}_{L-1}^{B} \in\left[H_{t}-R_{k} T_{c}, H_{t}\right)}}\left(\begin{array}{c}
L-1 \\
n_{1}, n_{2}, \ldots, n_{N}
\end{array}\right) \prod_{i=1}^{N} \pi_{i}^{n_{i}}\right) \pi_{k} .
\end{aligned}
$$

The inner sum in Eq. (11) is carried out over all possible $\overrightarrow{\mathbf{n}}$ 's satisfying that $\mathbb{H}_{L-1}^{B}$ falls into region $\left[H_{t}-R_{k} T_{c}, H_{t}\right]$.

\section{Transmission Time Analysis for Markov CHANNEL}

In this section, we derive the PDF of transmission time for big data transmission over Markov channels. We assume that the wireless channel can be modeled as a homogeneous continuous-time finite-state Markov chain. We adopt an $N$ state Markov chain with the $i$ th state corresponding to the event that the received SNR falls in $A_{i}, i=1,2, \cdots, N$, as illustrated in Fig. 3. The sojourn time of the Markov chain in state $i$ is an exponential random variable with average $\lambda_{i}$ calculated as [26]

$$
\lambda_{i}=\frac{\pi_{i}}{\operatorname{lcr}_{i}+\operatorname{lcr}_{i-1}} \quad i=1,2, \cdots, N .
$$


where $\operatorname{lcr}_{i}$ denotes the average level crossing rate with respect to boundary threshold $\gamma_{i}$. The PDF of the sojourn time in state $i$ is given by

$$
f_{T_{i}}(t)=\frac{1}{\lambda_{i}} \exp \left(-\frac{t}{\lambda_{i}}\right) .
$$

The transition rate from state $i$ to its neighbouring states, denoted by $q_{i-1 \mid i}$ and $q_{i+1 \mid i}$, can be respectively calculated as

$$
q_{i-1 \mid i}=\frac{\operatorname{lcr}_{i-1}}{\pi_{i}}, \quad q_{i+1 \mid i}=\frac{\mathrm{lcr}_{i}}{\pi_{i}} .
$$

It follows that the transition probability from state $i$ to state $i-1$ and $i+1$ are calculated as

$$
\begin{aligned}
& p_{i-1 \mid i}=\frac{q_{i-1 \mid i}}{q_{i-1 \mid i}+q_{i+1 \mid i}}=\frac{\operatorname{lcr}_{i-1}}{\operatorname{lcr}_{i-1}+\operatorname{lcr}_{i}}, \\
& p_{i+1 \mid i}=\frac{q_{i+1 \mid i}}{q_{i-1 \mid i}+q_{i+1 \mid i}}=\frac{\operatorname{lcr}_{i}}{\operatorname{lcr}_{i-1}+\operatorname{lcr}_{i}},
\end{aligned}
$$

respectively.

To implement $\mathrm{AMC}$, the receiver feeds back the index of the new AMC scheme only when the channel moves to a new state. The data rate is invariant while the channel remains in the same state. Transmission time for a large amount of data over Markov channels, denoted by $T_{\text {tr }}^{M}$, is equal to the sum of a sequence of random-length slots (See Fig. 4). The CDF of $T_{\mathrm{tr}}^{M}$ for a given amount of data $H_{t}$ is formulated, with the application of total probability theorem, as

$$
F_{\mathrm{tr}}^{M}(t)=\sum_{L} \operatorname{Pr}\left[T_{\mathrm{tr}, L}^{M}<t ; L \text { slots used }\right],
$$

where $T_{\operatorname{tr}, L}^{M}$ denotes the total transmission time when $L$ transmission slots are required to finish transmission and is in general given by

$$
T_{\mathrm{tr}, L}^{M}=\sum_{i=1}^{L-1} T^{(i)}+\frac{H_{t}-\sum_{i=1}^{L-1} R^{(i)} T^{(i)}}{R^{(L)}} .
$$

Here $R^{(i)}$ denotes the transmission rate over the $i$ th transmission slot and $T^{(i)}$ the duration of the $i$ th slot, which are modeled as independent but not identically distributed exponential random variables for Markov channels. Note that $L$ transmission slots are required to finish transmission if and only if the data transmitted over first $L-1$ transmission slots is less than $H_{t}$ and that transmitted over $L$ slots is larger than $H_{t}$. Therefore, the joint probability $\operatorname{Pr}\left[T_{\mathrm{tr}, L}^{M}<t ; L\right.$ slots used], denoted by $F_{\operatorname{tr}, L}^{M}(t)$, can be in general rewritten as

$$
\begin{aligned}
& F_{\mathrm{tr}, L}^{M}(t)=\operatorname{Pr}\left[\sum_{i=1}^{L-1} T^{(i)}+\frac{H_{t}-\sum_{i=1}^{L-1} R^{(i)} T^{(i)}}{R^{(L)}} \leq t,\right. \\
& \left.\sum_{i=1}^{L-1} R^{(i)} T^{(i)}<H_{t}, \sum_{i=1}^{L-1} R^{(i)} T^{(i)}+R^{(L)} T^{(L)} \geq H_{t}\right] .
\end{aligned}
$$

The corresponding PDF of $T_{\mathrm{tr}}^{M}$ is given by

$$
f_{\mathrm{tr}}^{M}(t)=\sum_{L} \frac{d}{d t} F_{\mathrm{tr}, L}^{M}(t)=\sum_{L} f_{\mathrm{tr}, L}^{M}(t) .
$$

We now derive the PDF/PMF of the transmission time when $L$ transmission slots are used, denoted by $f_{\mathrm{tr}, L}^{M}(t)$, for slow fading and fast fading scenarios separately.

\section{A. Slow Fading Scenario}

When the channel fading is slow and the amount of data transmitted in one transmission slot is larger than $H_{t}$, only one transmission slot is needed. The transmission time $T_{\mathrm{tr}, 1}^{M}$ is equal to $\frac{H_{t}}{R^{(1)}}$, if $R^{(1)}$ is used. As such, $T_{\mathrm{tr}, 1}^{M}$ is a discrete random variable with PMF calculated as

$$
\operatorname{Pr}\left[T_{\mathrm{tr}, 1}^{M}=\frac{H_{t}}{R^{(1)}}\right]=\operatorname{Pr}\left[T^{(1)} R^{(1)} \geq H_{t}\right] .
$$

Conditioning on that the received SNR falls into $A_{i}$, we have $R^{(1)}=R_{i}$ and $T^{(1)}$ is an exponential random variable with average $\lambda_{i}$. The PMF can be determined as

$$
\operatorname{Pr}\left[T_{\mathrm{tr}, 1}^{M}=\frac{H_{t}}{R_{i}}\right]=\pi_{i} e^{-\frac{H_{t}}{R_{i} \lambda_{i}}} .
$$

The overall PMF can be written in a PDF form as

$$
f_{\mathrm{tr}, 1}^{M}(t)=\sum_{i=1}^{N} \pi_{i} e^{-\frac{H_{t}}{R_{i} \lambda_{i}}} \delta\left(t-\frac{H_{t}}{R_{i}}\right),
$$

where $\delta(\cdot)$ is the unit impulse function.

If transmission completes in two slots, transmission time can be calculated as $T_{\mathrm{tr}, 2}^{M}=T^{(1)}+\frac{H_{t}-R^{(1)} T^{(1)}}{R^{(2)}}$. Accordingly, the CDF of $T_{\mathrm{tr}, 2}^{M}$ is formulated as the joint probability of three events

$$
\begin{aligned}
& F_{\mathrm{tr}, 2}^{M}(t)=\operatorname{Pr}\left[\left(1-\frac{R^{(1)}}{R^{(2)}}\right) T^{(1)}+\frac{H_{t}}{R^{(2)}} \leq t,\right. \\
& \left.R^{(1)} T^{(1)}<H_{t}, \quad R^{(1)} T^{(1)}+R^{(2)} T^{(2)} \geq H_{t}\right] .
\end{aligned}
$$

We proceed by conditioning on the channel realization and rewrite $T_{\mathrm{tr}, 2}^{M}$ as

$$
\begin{gathered}
F_{\mathrm{tr}, 2}^{M}(t)=\sum_{i=1}^{N}\left\{\operatorname { P r } \left[\left(R_{i+1}-R_{i}\right) T^{(1)} \leq t R_{i+1}-H_{t},\right.\right. \\
\left.R_{i} T^{(1)}<H_{t}, \quad R_{i} T^{(1)}+R_{i+1} T^{(2)} \geq H_{t}\right] p_{i+1 \mid i} \\
\quad+\operatorname{Pr}\left[\left(R_{i-1}-R_{i}\right) T^{(1)} \leq t R_{i-1}-H_{t},\right. \\
\left.\left.R_{i} T^{(1)}<H_{t}, \quad R_{i} T^{(1)}+R_{i-1} T^{(2)} \geq H_{t}\right] p_{i-1 \mid i}\right\} \pi_{i} .
\end{gathered}
$$

The first joint probability, denoted by $F^{+}(t)$, can be further rewritten as

$$
\begin{aligned}
F^{+}(t)=\operatorname{Pr}\left[T^{(1)}\right. & \leq \frac{t R_{i+1}-H_{t}}{R_{i+1}-R_{i}} \\
& \left.T^{(1)}<\frac{H_{t}}{R_{i}}, T^{(2)} \geq \frac{H_{t}-R_{i} T^{(1)}}{R_{i+1}}\right] .
\end{aligned}
$$

Note that given the channel was in state $i$ in the first transmission slot and then transit to state $i+1$ in the second slot, the duration of the first slot $T^{(1)}$ will be an exponential random variable with average $\lambda_{i}$ and that of the second slot $T^{(2)}$ will be another exponential random variable with average $\lambda_{i+1}$. 
Therefore, applying the PDFs of $T^{(1)}$ and $T^{(2)}, F^{+}(t)$ can be calculated as

$$
\begin{aligned}
& F^{+}(t)= \\
& \left\{\begin{array}{l}
\int_{0}^{\frac{t R_{i+1}-H_{t}}{R_{i+1}-R_{i}}} \int_{\frac{H_{t}-R_{i} x}{R_{i+1}}}^{\infty} f_{T_{i}}(x) f_{T_{i+1}}(y) d x \\
\int_{0}^{\frac{H_{t}}{R_{i}}} \int_{\frac{H_{t}-R_{i} x}{R_{i+1}}}^{\infty} f_{T_{i}}(x) f_{T_{i+1}}(y) d x d y
\end{array}\right. \\
& t \leq \frac{H_{t}}{R_{i+1}} \\
& 0 \\
& = \begin{cases}\frac{R_{i+1} \lambda_{i+1}}{R_{i} \lambda_{i}-R_{i+1} \lambda_{i+1}} e^{-\frac{H_{t}}{R_{i+1} \lambda_{i+1}}} & \\
\times\left[e^{\frac{\left(R_{i} \lambda_{i}-R_{i+1} \lambda_{i+1}\right)\left(t R_{i+1}-H_{t}\right)}{R_{i+1} \lambda_{i+1} \lambda_{i}\left(R_{i+1}-R_{i}\right.}}-1\right], t \in\left(\frac{H_{t}}{R_{i+1}}, \frac{H_{t}}{R_{i}}\right] \\
\frac{R_{i+1} \lambda_{i+1}}{R_{i} \lambda_{i}-R_{i+1} \lambda_{i+1}} e^{-\frac{H_{t}}{R_{i+1} \lambda_{i+1}}} & t>\frac{H_{t}}{R_{i}} . \\
\times\left[e^{\frac{\left(R_{i} \lambda_{i}-R_{i+1} \lambda_{i+1}\right) H_{t}}{R_{i+1} \lambda_{i+1} R_{i} \lambda_{i}}}-1\right], & \end{cases} \\
& t \in\left(\frac{H_{t}}{R_{i+1}}, \frac{H_{t}}{R_{i}}\right] \\
& t>\frac{H_{t}}{R_{i}} \\
& t \leq \frac{H_{t}}{R_{i+1}} ;
\end{aligned}
$$

Similarly, the other joint probability denoted by $F^{-}(t)$ can be calculated, while noting that $T^{(2)}$ will be an exponential random variables with average $\lambda_{i-1}$, as

$$
\begin{aligned}
& F^{-}(t)= \\
& \left\{\begin{array}{r}
0, \quad t \leq \frac{H_{t}}{R_{i}} ; \\
\frac{R_{i-1} \lambda_{i-1}}{R_{i} \lambda_{i}-R_{i-1} \lambda_{i-1}} e^{-\frac{H_{t}}{R_{i-1} \lambda_{i-1}}} \\
\times\left[e^{\frac{\left(R_{i} \lambda_{i}-R_{i-1} \lambda_{i-1}\right) H_{t}}{R_{i} \lambda_{i} R_{i-1} \lambda_{i-1}}}-e^{\left.\frac{\left(R_{i} \lambda_{i}-R_{i-1} \lambda_{i-1}\right)\left(t R_{i-1}-H_{t}\right)}{R_{i-1} \lambda_{i-1} \lambda_{i}\left(R_{i-1}-R_{i}\right)}\right]},\right. \\
\frac{t \in\left(\frac{H_{t}}{R_{i}}, \frac{H_{t}}{R_{i-1}}\right] ;}{R_{i-1} \lambda_{i-1}} e^{-\frac{H_{t}}{R_{i-1} \lambda_{i-1}}}\left[e^{\left.\frac{\left(R_{i} \lambda_{i}-R_{i-1} \lambda_{i-1}\right) H_{t}}{R_{i-1} \lambda_{i-1} R_{i} \lambda_{i}}-1\right]}\right. \\
t>\frac{H_{t}}{R_{i-1}} .
\end{array},\right.
\end{aligned}
$$

After substituting Eq. (26) and Eq. (27) into Eq. (24) and taking derivative with respect to $t$, the PDF of transmission time when two transmission slots is needed to finish data transmission is given by

$$
\begin{aligned}
& f_{\mathrm{tr}, 2}^{M}(t)= \\
& \sum_{i=1}^{N}\left\{\frac{R_{i+1}}{\lambda_{i}\left(R_{i+1}-R_{i}\right)} e^{-\frac{H_{t}}{R_{i+1} \lambda_{i+1}}} e^{\frac{\left(R_{i} \lambda_{i}-R_{i+1} \lambda_{i+1}\right)\left(t R_{i+1}-H_{t}\right)}{R_{i+1} \lambda_{i+1} \lambda_{i}\left(R_{i+1}-R_{i}\right)}}\right. \\
& \times\left[\mathcal{U}\left(t-\frac{H_{t}}{R_{i+1}}\right)-\mathcal{U}\left(t-\frac{H_{t}}{R_{i}}\right)\right] p_{i+1 \mid i} \\
& +\frac{R_{i-1}}{\lambda_{i}\left(R_{i}-R_{i-1}\right)} e^{-\frac{H_{t}}{R_{i-1} \lambda_{i-1}}} e^{\frac{\left(R_{i} \lambda_{i}-R_{i-1} \lambda_{i-1}\right)\left(t R_{i-1}-H_{t}\right)}{R_{i-1} \lambda_{i-1} \lambda_{i}\left(R_{i-1}-R_{i}\right)}} \\
& \left.\times\left[\mathcal{U}\left(t-\frac{H_{t}}{R_{i}}\right)-\mathcal{U}\left(t-\frac{H_{t}}{R_{i-1}}\right)\right] p_{i-1 \mid i}\right\} \pi_{i} .
\end{aligned}
$$

The number of transmission slots depends on the average length of each slot $\lambda_{i}, i=1,2 \cdots, N$, the amount of data to be transmitted $H_{t}$, and transmission rate used $R_{i}, i=$ $1,2 \cdots, N$. If the channel introduces slow fading and the transmission rate is high, transmission will most likely complete over two time slots. For example, for slow fading environment, $\lambda_{i}$ may have average values of several seconds. If the transmission rate $R_{i}$ are in the order of Gbps, then the system can transmit several Giga bits over a few slots. Therefore, the overall PDF of the transmission time for slow fading environment can be approximated by $f_{\mathrm{tr}, 1}^{M}(t)+f_{\mathrm{tr}, 2}^{M}(t)$.

\section{B. Fast Fading Scenario}

For the fast fading case, the number of transmission slots required to finish data transmission can be very large. In this case, the CDF of $T_{\mathrm{tr}, L}^{M}$, denoted by $F_{\mathrm{tr}, L}^{M}(t)$, is generally formulated as

$$
\begin{aligned}
F_{\mathrm{tr}, L}^{M}(t)=\operatorname{Pr} & {\left[\mathbb{T}_{L-1}^{M}+\frac{H_{t}-\mathbb{H}_{L-1}^{M}}{R^{(L)}} \leq t,\right.} \\
& \left.\mathbb{H}_{L-1}^{M}<H_{t}, \mathbb{H}_{L-1}^{M}+R^{(L)} T^{(L)} \geq H_{t}\right],
\end{aligned}
$$

where $\mathbb{T}_{L-1}^{M}=\sum_{i=1}^{L-1} T^{(i)}$ denotes the time duration of first $L-1$ transmission slots and $\mathbb{H}_{L-1}^{M}=\sum_{i=1}^{L-1} R^{(i)} T^{(i)}$ denotes the amount of data transmitted over the first $L-1$ slots. To proceed further, we again condition on the channel realization. Let us consider a particular channel realization over $L$ transmission slots, where the channel stays in state $k$ in the $L$ th slot. As such, $R^{(L)}$ will be equal to $R_{k}$ based on the mode of operation and $T^{(L)}$ will be an exponential random variable with mean $\lambda_{k}$. Suppose also that rate $R_{j}$ used $n_{j}$ times over the first $L-1$ slots, where $\sum_{j=1}^{N} n_{j}=L-1$. Then, the time duration of first $L-1$ transmission slots $\mathbb{T}_{L-1}^{M}$ will be the sum of $n_{1}$ random variables with PDF $f_{T_{1}}(t), n_{2}$ random variables with PDF $f_{T_{2}}(t), \ldots$, and $n_{N}$ random variables with PDF $f_{T_{N}}(t)$. Furthermore, $\mathbb{H}_{L-1}^{M}$ will be the sum of $n_{1}$ random variables with PDF $f_{T_{1}}\left(t / R_{1}\right) / R_{1}, n_{2}$ random variables with PDF $f_{T_{2}}\left(t / R_{2}\right) / R_{2}, \ldots$, and $n_{N}$ random variables with PDF $f_{T_{N}}\left(t / R_{N}\right) / R_{N}$. These relationships apply to all channel realizations that leads to the same vector $\overrightarrow{\mathbf{n}}=\left[n_{1}, n_{2}, \cdots, n_{N}\right]$ and the same channel state in $L$ th slot. By conditioning on the channel realization leading to the same $\overrightarrow{\mathbf{n}}$ and $L$ th slot state, we arrive at Eq. (30) shown at the top of this page. 


$$
\begin{aligned}
& F_{\mathrm{tr}, L}^{M}(t)=\sum_{k=1}^{N} \sum_{\overrightarrow{\mathbf{n}}} \operatorname{Pr}\left[\mathbb{T}_{L-1 \mid \overrightarrow{\mathbf{n}}}^{M}+\frac{H_{t}-\mathbb{H}_{L-1 \mid \overrightarrow{\mathbf{n}}}^{M}}{R_{k}} \leq t, \mathbb{H}_{L-1 \mid \overrightarrow{\mathbf{n}}}^{M}<H_{t}, \mathbb{H}_{L-1 \mid \overrightarrow{\mathbf{n}}}^{M}+R_{k} T^{(L)} \geq H_{t}\right] \\
& \times \operatorname{Pr}[\overrightarrow{\mathbf{n}} \& \text { slot } L \text { in state } k] \\
& f_{\mathbb{T}_{L-1 \mid \overrightarrow{\mathbf{n}}}^{M}, \mathbb{H}_{L-1 \mid \overrightarrow{\mathbf{n}}}^{M}}(x, y)=\frac{1}{2 \pi \sigma_{x} \sigma_{y} \sqrt{1-\rho^{2}}} \exp \left(-\frac{1}{2\left(1-\rho^{2}\right)}\left(\frac{\left(x-\eta_{x}\right)^{2}}{\sigma_{x}{ }^{2}}-2 \rho \frac{\left(x-\eta_{x}\right)\left(y-\eta_{y}\right)}{\sigma_{x} \sigma_{y}}+\frac{\left(y-\eta_{y}\right)^{2}}{\sigma_{y}{ }^{2}}\right)\right) \\
& f_{\mathrm{tr}, L \mid \overrightarrow{\mathbf{n}}, k}^{M}(t)=\frac{\exp \left(-\frac{H_{t}}{R_{k}}\right) \exp \left[-\frac{\left(t-\frac{H_{t}}{R_{k}}-\eta_{x}\right)^{2} \sigma_{y}{ }^{2}+2 \rho \eta_{y} \sigma_{x} \sigma_{y}\left(t-\frac{H_{t}}{R_{k}}-\eta_{x}\right)+\sigma_{x}{ }^{2} \eta_{y}{ }^{2}}{2\left(1-\rho^{2}\right) \sigma_{x}{ }^{2} \sigma_{y}{ }^{2}}\right]}{4 \pi \sigma_{x} \sigma_{y} \sqrt{1-\rho^{2}}} \sqrt{\frac{2 \pi\left(1-\rho^{2}\right) \sigma_{x}{ }^{2} \sigma_{y}{ }^{2}}{\left(\frac{\sigma_{y}{ }^{2}}{R_{k}^{2}}-2 \rho \frac{\sigma_{x} \sigma_{y}}{R_{k}}+\sigma_{x}{ }^{2}\right)}} \\
& \times \exp \left\{\frac{\left[\frac{\left(t-\frac{H_{t}}{R_{k}}-\eta_{x}\right) \sigma_{y}{ }^{2}+\rho \eta_{y} \sigma_{x} \sigma_{y}}{R_{k}}-\frac{\left(1-\rho^{2}\right) \sigma_{x}{ }^{2} \sigma_{y}{ }^{2}}{R_{k} \lambda_{k}}-\rho \sigma_{x} \sigma_{y}\left(t-\frac{H_{t}}{R_{k}}-\eta_{x}\right)-\sigma_{x}{ }^{2} \eta_{y}\right]^{2}}{\left(\frac{\sigma_{y}{ }^{2}}{R_{k}^{2}}-2 \rho \frac{\sigma_{x} \sigma_{y}}{R_{k}}+\sigma_{x}{ }^{2}\right) 2\left(1-\rho^{2}\right) \sigma_{x}{ }^{2} \sigma_{y}{ }^{2}}\right\} \\
& \times \operatorname{erf}\left[\frac{\left(\frac{\left(t-\frac{H_{t}}{R_{k}}-\eta_{x}\right) \sigma_{y}{ }^{2}+\rho \eta_{y} \sigma_{x} \sigma_{y}}{R_{k}}-\frac{\left(1-\rho^{2}\right) \sigma_{x}{ }^{2} \sigma_{y}{ }^{2}}{R_{k} \lambda_{k}}-\rho \sigma_{x} \sigma_{y}\left(t-\frac{H_{t}}{R_{k}}-\eta_{x}\right)-\sigma_{x}{ }^{2} \eta_{y}\right)}{\sqrt{\left(\frac{\sigma_{y}{ }^{2}}{R_{k}^{2}}-2 \rho \frac{\sigma_{x} \sigma_{y}}{R_{k}}+\sigma_{x}{ }^{2}\right) 2\left(1-\rho^{2}\right) \sigma_{x}{ }^{2} \sigma_{y}{ }^{2}}}+\sqrt{\frac{2\left(1-\rho^{2}\right) \sigma_{x}{ }^{2} \sigma_{y}{ }^{2}}{\left(\frac{\sigma_{y}^{2}}{R_{k}^{2}}-2 \rho \frac{\sigma_{x} \sigma_{y}}{R_{k}}+\sigma_{x}{ }^{2}\right)}} H_{t}\right] \\
& \left.-\operatorname{erf}\left(\frac{\left(\frac{\left(t-\frac{H_{t}}{R_{k}}-\eta_{x}\right) \sigma_{y}{ }^{2}+\rho \eta_{y} \sigma_{x} \sigma_{y}}{R_{k}}-\frac{\left(1-\rho^{2}\right) \sigma_{x}{ }^{2} \sigma_{y}{ }^{2}}{R_{k} \lambda_{k}}-\rho \sigma_{x} \sigma_{y}\left(t-\frac{H_{t}}{R_{k}}-\eta_{x}\right)-\sigma_{x}^{2} \eta_{y}\right)}{\sqrt{\left(\frac{\sigma_{y}^{2}}{R_{k}^{2}}-2 \rho \frac{\sigma_{x} \sigma_{y}}{R_{k}}+\sigma_{x}^{2}\right) 2\left(1-\rho^{2}\right) \sigma_{x}{ }^{2} \sigma_{y}{ }^{2}}}\right)\right\}
\end{aligned}
$$

Let $F_{\mathrm{tr}, L \mid \overrightarrow{\mathbf{n}}, k}^{M}(t)$ denote $\operatorname{Pr}\left[\mathbb{T}_{L-1 \mid \overrightarrow{\mathbf{n}}}^{M}+\frac{H_{t}-\mathbb{H}_{L-1 \mid \overrightarrow{\mathbf{n}}}^{M}}{R_{k}} \leq\right.$ $\left.t, \mathbb{H}_{L-1 \mid \overrightarrow{\mathbf{n}}}^{M}<H_{t}, \mathbb{H}_{L-1 \mid \overrightarrow{\mathbf{n}}}^{M}+R_{k} T^{(L)} \geq H_{t}\right]$. In general, calculating $F_{\mathrm{tr}, L \mid \overrightarrow{\mathbf{n}}, k}^{M}(t)$ is challenging since it is the joint probability involving correlated events. Essentially, $\mathbb{T}_{L-1 \mid \overrightarrow{\mathbf{n}}}^{M}=$ $\sum_{i=1}^{L-1} T^{(i)}$ and $\mathbb{H}_{L-1 \mid \overrightarrow{\mathbf{n}}}^{M}=\sum_{i=1}^{L-1} R^{(i)} T^{(i)}$ are two linear combinations of one identical set of $L-1$ i.n.d. exponential random variables, whose joint PDF is unknown even in the statistic literature, to the best of authors' knowledge. To proceed further, assuming a large number of transmission slots are needed to complete transmission, especially for fast fading case, we apply central limit theory to obtain an approximate expression of $F_{\mathrm{tr}, L \mid \overrightarrow{\mathbf{n}}, k}^{M}(t)$ for moderately large value of $L$. Specifically, we approximate $\mathbb{T}_{L-1 \mid \overrightarrow{\mathbf{n}}}^{M}$ and $\mathbb{H}_{L-1 \mid \overrightarrow{\mathbf{n}}}^{M}$ as jointly Gaussian distributed random variables (i.e. $\left(\mathbb{T}_{L-1 \mid \mathbf{n}^{M}}^{M} \mathbb{H}_{L-1 \mid \overrightarrow{\mathbf{n}}}^{M}\right) \sim \mathcal{N}\left(\eta_{x}, \sigma_{x}^{2}, \eta_{y}, \sigma_{y}^{2}, \quad \rho\right)$ ), whose parameters are derived in Appendix A. The joint PDF of $\mathbb{T}_{L-1 \mid \overrightarrow{\mathbf{n}}}^{M}$ and $\mathbb{H}_{L-1 \mid \overrightarrow{\mathbf{n}}}^{M}$ is shown in Eq. (31) at the top of this page. By conditioning on the independent random variable $T^{(L)}, F_{\mathrm{tr}, L \mid \overrightarrow{\mathbf{n}}, k}^{M}(t)$ can now be calculated as

$$
\begin{aligned}
& F_{\mathrm{tr}, L \mid \overrightarrow{\mathbf{n}}, k}^{M}(t)= \\
& \int_{0}^{H_{t}} \int_{-\infty}^{t-\frac{H_{t}-y}{R_{k}}} f_{\mathbb{T}_{L-1 \mid \overrightarrow{\mathbf{n}}}^{M}, \mathbb{H}_{L-1 \mid \overrightarrow{\mathbf{n}}}^{M}}(x, y) e^{\left(-\frac{H_{t}-y}{\lambda_{k} R_{k}}\right)} d x d y .
\end{aligned}
$$

For Markov channel, the probability of all channel real- izations that lead to vector $\overrightarrow{\mathbf{n}}$ over first $L-1$ slots and state $k$ in the last slot, is challenging to calculate exactly, due to the correlation between channel state over subsequent transmission slots. In the following, we assume that the number of transmission slots is large and as such, the possible channel realizations lead to the same vector $\overrightarrow{\mathbf{n}}$ are huge. Then the joint probability $\operatorname{Pr}[\overrightarrow{\mathbf{n}} \&$ slot $L$ in state $k]$ can be approximately calculated by neglecting the correlation as

$$
\operatorname{Pr}[\overrightarrow{\mathbf{n}} \& \text { slot } L \text { in state } k]=\pi_{k}\left(\begin{array}{c}
L-1 \\
n_{1}, n_{2}, \cdots, n_{N}
\end{array}\right) \prod_{j=1}^{N} \pi_{j}^{n_{j}} .
$$

After substituting Eq. (31) and Eq. (33) into Eq. (30), taking derivative with respect to $t$ and much manipulation with the help of [27], we obtain the PDF of the transmission time when $L$ transmission slots are needed, $T_{\mathrm{tr}, L}^{M}$, as

$$
f_{\mathrm{tr}, L}^{M}(t)=\sum_{k=1}^{N} \sum_{\overrightarrow{\mathbf{n}}} f_{\mathrm{tr}, L \mid \overrightarrow{\mathbf{n}}, k}^{M}(t) \pi_{k}\left(\begin{array}{c}
L-1 \\
n_{1}, n_{2}, \cdots, n_{N}
\end{array}\right) \prod_{j=1}^{N} \pi_{j}^{n_{j}}
$$

where the analytical expression of $f_{\mathrm{tr}, L \mid \overrightarrow{\mathbf{n}}, k}^{M}(t)$ is given by Eq. (35) shown at the top of previous page, where $\operatorname{erf}(\cdot)$ is the Gauss error function.

Finally, the distribution function of transmission time, $T_{\mathrm{tr}}^{M}$, can be evaluated by substituting Eq. (34) into Eq. (19) and taking summation over an appropriate range of integer $L$. In 


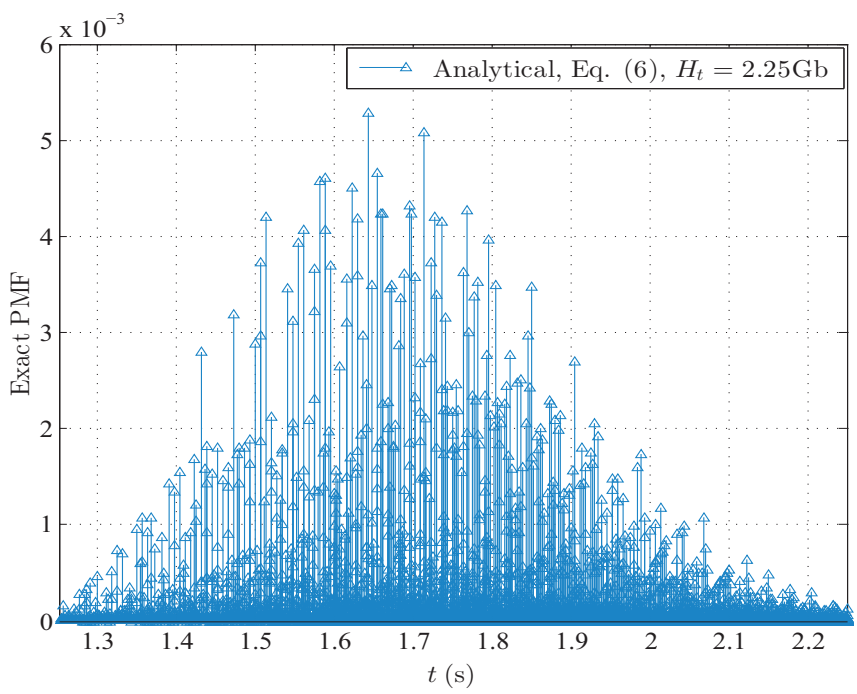

Fig. 5. Exact PMF of transmission time over block fading channel, where $T_{c}=80 \mathrm{~ms}, \bar{\gamma}=15 \mathrm{~dB}$.

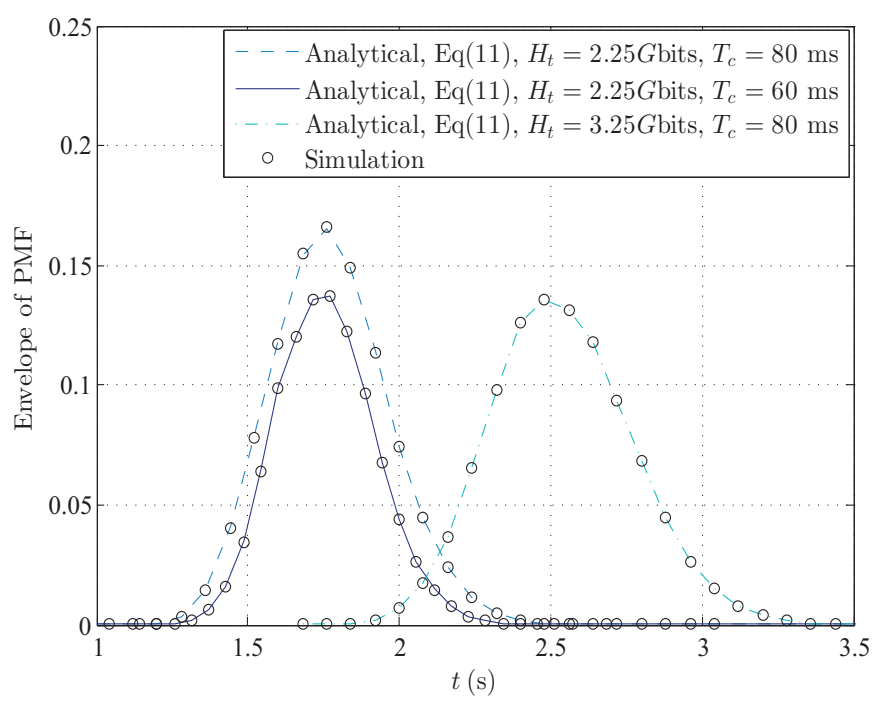

Fig. 6. Monte Carlo verification of analytical result of approximate PMF, where, $\bar{\gamma}=15 \mathrm{~dB}$.

fact, the average number of slots required can be estimated by $L_{\text {ave }}^{M}=\frac{H_{t}}{\sum_{i=1}^{N} R_{i} \lambda_{i} \pi_{i}}$. We can achieve satisfactory accuracy by taking summation over $L \in\left[1,\left\lfloor 3 L_{\text {ave }}^{M}\right\rfloor\right]$ as shown in numerical examples.

\section{Numerical Results}

In this section, we present selected numerical results to illustrate the mathematical formulations and verify the analytical approach. We adopt broadband video downloading, which accounts for more than half of mobile data traffic currently [28], as a target application. Specifically, the wireless system is transmitting a high-quality movie file with size in the order of Gbits. We assume a wideband transmission system with a channel bandwidth of $500 \mathrm{MHz}$, which supports a symbol rate of $500 \mathrm{Msps}$, applicable to millimeter wave

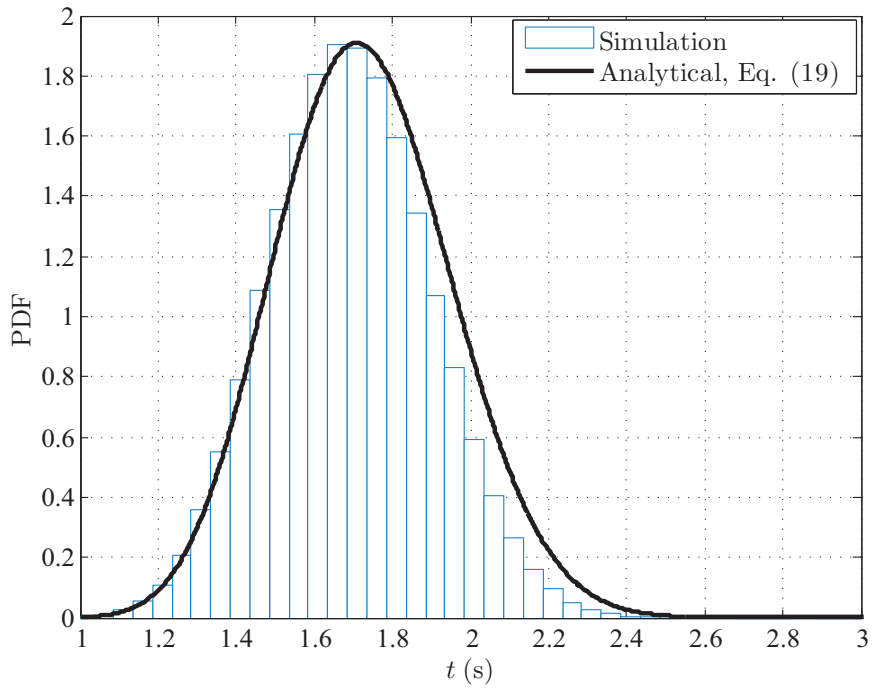

Fig. 8. Both Monte Carlo simulation and analytical result in evaluating $T_{\mathrm{tr}}^{M}$, where $H_{t}=3.25 \mathrm{~Gb}, f_{D}=5 \mathrm{~Hz}, \bar{\gamma}=15 \mathrm{~dB}$.

(mmWave) and ultra wide band (UWB) systems. The AMC scheme is adapted from LTE standard [29], where the value range of received SNR is divided into five regions. The first region corresponds to no transmission and the remaining four regions correspond to AMC transmission modes with date rate $\left[R_{1}, R_{2}, R_{3}, R_{4}\right]=[0.3008,0.975,1.9512,2.7773] \mathrm{Gbps}$, respectively. The SNR thresholds for transmission modes are set as $\left[\gamma_{1}, \gamma_{2}, \gamma_{3}, \gamma_{4}\right]=[-1.254,6.528,14.18,19.83] \mathrm{dB}$ to satisfy an instantaneous error rate requirement. We apply the Rayleigh fading model (i.e. $\gamma \sim \operatorname{Exp}(\bar{\gamma})$ ). The level crossing rate for Rayleigh fading can be calculated by

$$
\operatorname{lcr}_{i}=\sqrt{\frac{2 \pi \gamma_{i}}{\bar{\gamma}}} f_{D} \exp \left(-\frac{\gamma_{i}}{\bar{\gamma}}\right),
$$

where $\bar{\gamma}$ is the average received SNR and $f_{D}$ is the Doppler shift. The probability that transmission rate $R_{i}$ is used can be calculated by Eq. (1) accordingly.

Fig. 5 presents the exact PMF of the transmission time $T_{\mathrm{tr}}^{B}$ when transmitting a video file of size $2.25 \mathrm{~Gb}$ with AMC over block fading channel. We observe that, to complete transmission, multiple $T_{c}$ 's are needed. The transmission time $T_{\mathrm{tr}}^{B}$ varies dramatically around its average value. The distribution of $T_{\mathrm{tr}}^{B}$ depends on the average received SNR, the channel coherence time, and the amount of data. We examine the behavior of the distribution of $T_{\mathrm{tr}}^{B}$ in Fig. 6 by plotting the envelop of approximate PDF given in Eq. (11) with different parameter settings. The Monte Carlo simulation with $10^{8}$ samples are also presented as the black circles. We first observe that the analytical result matches the simulation result well. As such, the approximate solution can serve an effective alternative in $T_{\mathrm{tr}}^{B}$ analysis. As data amount is larger, it is intuitive that the average of $T_{\mathrm{tr}}^{B}$ increases. For longer channel coherence time, the variance of $T_{\text {tr }}^{B}$ increases since fewer $T_{c}$ 's are needed to complete transmission. However, given the data amount, the average value of $T_{\text {tr }}^{B}$ barely changes for longer $T_{c}$. 

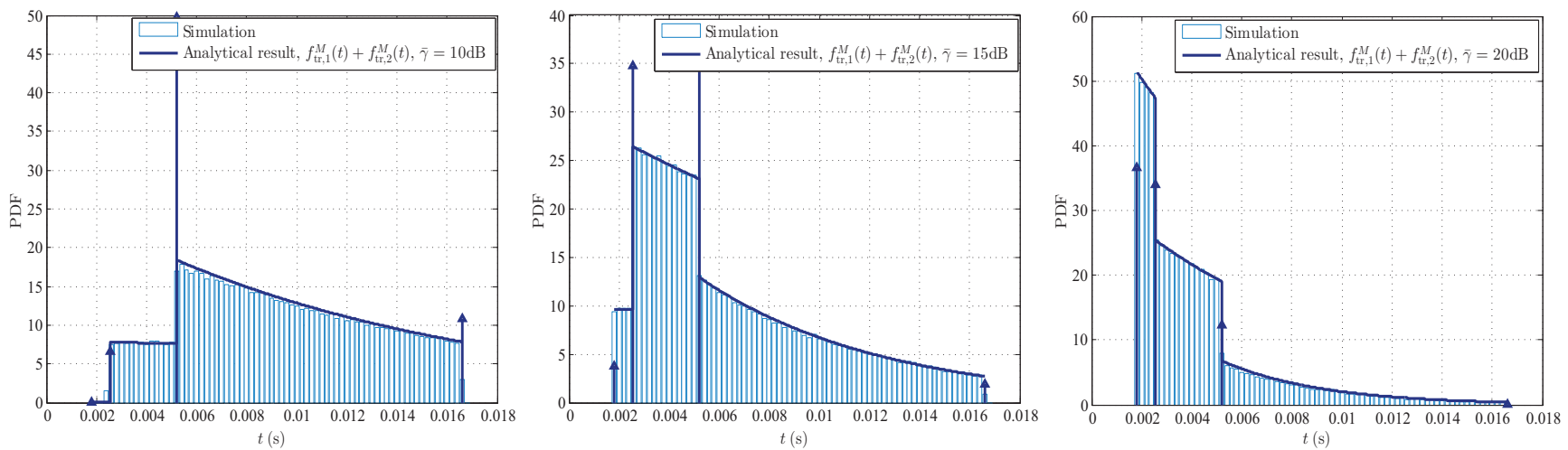

Fig. 7. Monte Carlo simulation verification of analytical expression of PDF for $T_{\mathrm{tr}}^{M}$ for transmitting relatively small amount of data with AMC over slow fading $\left(\right.$ e.g. $\left.f_{\mathrm{tr}}^{M}(t) \approx f_{\mathrm{tr}, 1}^{M}(t)+f_{\mathrm{tr}, 2}^{M}(t)\right)$, where $H_{t}=10 \mathrm{Mb}$, and $f_{D}=10 \mathrm{~Hz}$.

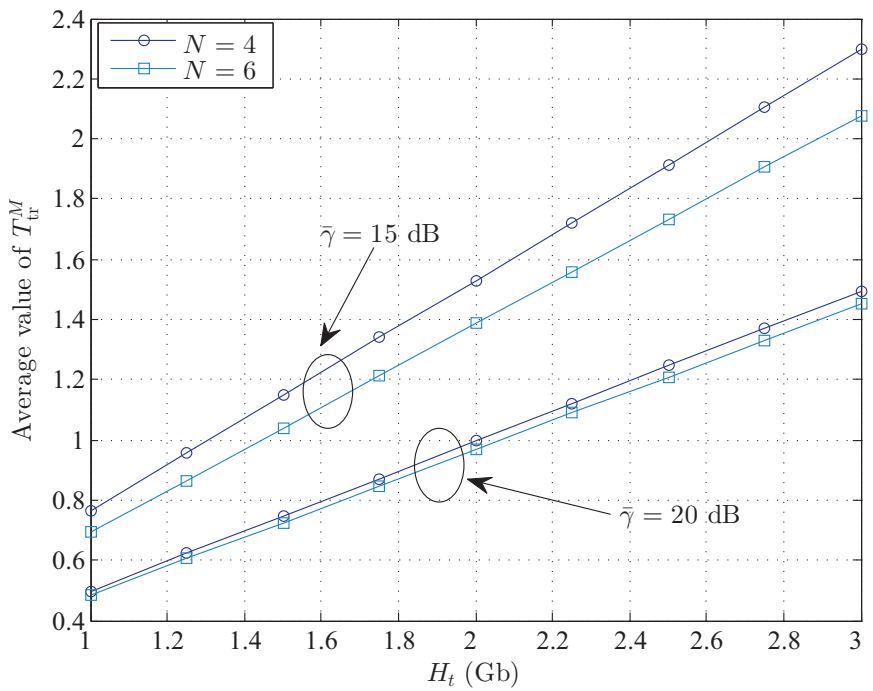

Fig. 9. Average value of transmission time over continuous-time Markov channel, where $f_{D}=5 \mathrm{~Hz}$.

Fig. 7 plots the analytical PDF of the transmission time $T_{\mathrm{tr}}^{M}$ for slow Markov fading scenario and corresponding simulation results versus various average received SNR. The perfect match between the analytical result and Monte Carlo simulation validates our analytical approach. We notice that $T_{\mathrm{tr}}^{M}$ can neither be greater than its maximum value $H_{t} / R_{1}$ nor less than its minimum value $H_{t} / R_{N}$, as expected. We observe that the probability distribution of $T_{\mathrm{tr}}^{M}$ over each subregion $\left[H_{t} / R_{i+1}, H_{t} / R_{i}\right]$ varies w.r.t. channel condition. The average value of $T_{\mathrm{tr}}^{M}$ approaches the minimum transmission time when average received SNR increases. Fig. 8 compares the analytical results for the PDF of $T_{\mathrm{tr}}^{M}$ given in Eq. (19) with the result obtained from Monte Carlo simulation. We notice that the analytical result slightly over-estimate the transmission time. The mismatch results from applying the central limit theory and ignoring the memory property of Markov channels. As the number of required channel coherence time $L$ gets larger, the approximation becomes more accurate.

Fig. 9 plots the mean of $T_{\mathrm{tr}}^{M}$ as a function of data amount with different average received SNR and two different AMC implementations. We can see that the average value of $T_{\text {tr }}^{M}$ increases with increasing $H_{t}$, as expected. When the average received SNR is larger, higher rate AMC schemes are more frequently used, resulting in smaller average value of $T_{\mathrm{tr}}^{M}$. To examine the effect of the number of AMC transmission rate, we introduce another AMC implementation, where two more transmission rates are used with $R_{5}=0.5879 \mathrm{Gbps}$ and $R_{6}=1.3653$ Gbps. Accordingly, the received SNR thresholds are $\gamma_{5}=2.7 \mathrm{~dB}$ and $\gamma_{6}=10.37 \mathrm{~dB}$, respectively. AMC implementation with more transmission rates can take advantage of relatively better channel condition, which reduces the average value of transmission time. However, when the average received SNR is large, higher rate AMC schemes are frequently used for both AMC implementations. As such, the improvement becomes less significant.

\section{CONCLUSION}

In this paper, we proposed an analytical approach to investigate the transmission time of big data with AMC over wireless channels. When the amount of data becomes massive, the transmission will experience variable channel quality, and as such, different transmission rate with AMC. We derived the exact PMF and CDF of the transmission time under block fading channel model. Then we generalized our analysis to continuous-time Markov channel, where transmission slots have random length. The distribution functions of the transmission time for slow and fast fading environments are obtained. While previous work assumes constant transmission time for a fixed amount of data, we observe that transmission time of big data varies drastically around its average.

Transmission time characterization for big data will be essential to the optimal design and performance analysis of future wireless transmission systems for big data applications. The queuing delay performance for wireless transmission of big data can be evaluated following similar steps in [8] with the first-order and second-order statistics of transmission time. With proper model for the blockage process of mmWave links, the analytical result of this work can directly apply to evaluate the impact of blockage on the throughput of mmWave system. On-going effort is to apply these statistical result to design 
energy efficient communication system. The effect of outdated channel state information (CSI) on the transmission time is another interesting future research direction.

\section{APPENDIX A}

\section{Derivation of Conditional CDF in $F_{\mathrm{tr}, L}(t)$}

$\mathbb{T}_{L-1 \mid \overrightarrow{\mathbf{n}}}^{M}=\sum_{i=1}^{L-1} T^{(i)}$ and $\mathbb{H}_{L-1 \mid \overrightarrow{\mathbf{n}}}^{M}=\sum_{i=1}^{L-1} R^{(i)} T^{(i)}$ are two linear combination of $L-1$ independently nonidentically distributed exponential random variables $T_{l}, l=$ $1, \cdots, L-1$. We approximate that $\mathbb{T}_{L-1 \mid \overrightarrow{\mathbf{n}}}^{M}$ and $\mathbb{H}_{L-1 \mid \overrightarrow{\mathbf{n}}}^{M}$ are jointly Gaussian distributed random variables. Specifically, $\left(\mathbb{T}_{L-1 \mid \overrightarrow{\mathbf{n}}}^{M} \mathbb{H}_{L-1 \mid \overrightarrow{\mathbf{n}}}^{M}\right) \sim \mathcal{N}\left(\eta_{x}, \sigma_{x}^{2}, \eta_{y}, \sigma_{y}^{2}, \rho\right)$. Given the channel realization, we can directly calculate their marginal first and second order statistics as $\eta_{x}=\mathbb{E}\left[\mathbb{T}_{L-1 \mid \overrightarrow{\mathbf{n}}}^{M}\right]=\sum_{i=1}^{N} n_{i} \lambda_{i}$, $\sigma_{x}^{2}=\mathbb{V}\left[\mathbb{T}_{L-1 \mid \overrightarrow{\mathbf{n}}}^{M}\right]=\sum_{i=1}^{N} n_{i} \lambda_{i}^{2}, \eta_{y}=\mathbb{E}\left[\mathbb{H}_{L-1 \mid \overrightarrow{\mathbf{n}}}^{M}\right]=$ $\sum_{i=1}^{N} n_{i} R_{i} \lambda_{i}$ and $\sigma_{y}^{2}=\mathbb{V}\left[\mathbb{H}_{L-1 \mid \overrightarrow{\mathbf{n}}}^{M}\right]=\sum_{i=1}^{N} n_{i}\left(R_{i} \lambda_{i}\right)^{2}$, where $\mathbb{E}[\cdot]$ and $\mathbb{V}[\cdot]$ denote the expectation and variance, respectively. The covariance of $\mathbb{T}_{L-1 \mid \overrightarrow{\mathbf{n}}}^{M}$ and $\mathbb{H}_{L-1 \mid \overrightarrow{\mathbf{n}}}^{M}$, denoted by $\mathbb{C}_{\mathbb{T}_{L-1 \mid \overrightarrow{\mathbf{n}}}^{M} \mathbb{H}_{L-1 \mid \overrightarrow{\mathbf{n}}}^{M}}$, can be calculated as

$$
\begin{aligned}
\mathbb{C}_{\mathbb{T}_{L-1 \mid \overrightarrow{\mathbf{n}}}^{M} \mathbb{H}_{L-1 \mid \overrightarrow{\mathbf{n}}}^{M}} & =\mathbb{E}\left[\mathbb{T}_{L-1 \mid \overrightarrow{\mathbf{n}}}^{M} \mathbb{H}_{L-1 \mid \overrightarrow{\mathbf{n}}}^{M}\right]-\mathbb{E}\left[\mathbb{T}_{L-1 \mid \overrightarrow{\mathbf{n}}]}^{M}\right] \mathbb{E}\left[\mathbb{H}_{L-1 \mid \overrightarrow{\mathbf{n}}}^{M}\right] \\
= & \mathbb{E}\left[\left(\sum_{l=1}^{L-1} T^{(l)}\right)\left(\sum_{j=1}^{L-1} R^{(j)} T^{(j)}\right)\right] \\
= & -\mathbb{E}\left[\sum_{l=1}^{L-1} T^{(l)}\right] \mathbb{E}\left[\sum_{j=1}^{L-1} R^{(j)} T^{(j)}\right] \\
& \mathbb{E}\left[\sum_{l=1}^{L-1} \sum_{j=1}^{L-1} R^{(j)} T^{(j)} T^{(l)}\right] \\
= & \left.\sum_{l=1}^{L-1} \sum_{j=1}^{L-1} R^{(j)} \mathbb{E}\left[T^{(l)}\right]\right)\left(\sum_{j=1}^{L-1} \mathbb{E}\left[R^{(j)} T^{(j)}\right]\right)
\end{aligned}
$$

If the number of transmission slots is large and as such, the possible channel realizations lead to the same vector $\overrightarrow{\mathbf{n}}$ are huge. By neglecting the correlation, $T^{(l)}$ and $T^{(j)}$ are independent when $l \neq j$, as such $\mathbb{E}\left[T^{(l)} T^{(j)}\right]-\mathbb{E}\left[T^{(l)}\right] \mathbb{E}\left[T^{(j)}\right]=0$ for $l \neq j$. Therefore, $\mathbb{C}_{\mathbb{T}_{L-1 \mid \overrightarrow{\mathbf{n}}}^{M} \mathbb{H}_{L-1 \mid \overrightarrow{\mathbf{n}}}^{M}}$ can be rewritten as

$$
\begin{aligned}
\mathbb{C}_{\mathbb{T}_{L-1 \mid \overrightarrow{\mathbf{n}}}^{M} \mathbb{H}_{L-1 \mid \overrightarrow{\mathbf{n}}}^{M}} & =\sum_{l=1}^{L-1} R^{(l)} \mathbb{E}\left[\left(T^{(l)}\right)^{2}\right]-\sum_{l=1}^{L-1} R^{(l)} \mathbb{E}^{2}\left[T^{(l)}\right] \\
& =\sum_{l=1}^{L-1} R^{(l)}\left(\mathbb{E}\left[\left(T^{(l)}\right)^{2}\right]-\mathbb{E}^{2}\left[T^{(l)}\right]\right) \\
& =\sum_{l=1}^{L-1} R^{(l)} \mathbb{V}\left[T^{(l)}\right]=\sum_{i=1}^{N} n_{i} R_{i} \lambda_{i}^{2} .
\end{aligned}
$$

Hence, by definition, the correlation coefficient can be calculated as

$$
\begin{aligned}
\rho & =\frac{\mathbb{C}_{\mathbb{T}_{L-1 \mid \overrightarrow{\mathbf{n}}}^{M}} \mathbb{H}_{L-1 \mid \overrightarrow{\mathbf{n}}}^{M}}{\sqrt{\mathbb{V}\left[\mathbb{T}_{L-1 \mid \overrightarrow{\mathbf{n}}}^{M}\right]} \sqrt{\mathbb{V}\left[\mathbb{H}_{L-1 \mid \overrightarrow{\mathbf{n}}}^{M}\right]}} \\
& =\frac{\sum_{i=1}^{N} n_{i} R_{i} \lambda_{i}^{2}}{\sqrt{\sum_{i=1}^{N} n_{i} \lambda_{i}^{2}} \sqrt{\sum_{i=1}^{N} n_{i}\left(R_{i} \lambda_{i}\right)^{2}}}
\end{aligned}
$$

As such, $F_{\operatorname{tr}, L \mid \overrightarrow{\mathbf{n}}, k}^{M}(t)$ can be rewritten as

$$
\begin{aligned}
F_{\mathrm{tr}, L \mid \overrightarrow{\mathbf{n}}, k}^{M}(t) & =\operatorname{Pr}\left[\mathbb{T}_{L-1 \mid \overrightarrow{\mathbf{n}}}^{M} \leq t-\frac{H_{t}-\mathbb{H}_{L-1 \mid \overrightarrow{\mathbf{n}}}^{M}}{R_{k}} ;\right. \\
0 & \left.\leq \mathbb{H}_{L-1 \mid \overrightarrow{\mathbf{n}}}^{M} \leq H_{t} ; T^{(L)} \geq \frac{H_{t}-\mathbb{H}_{L-1 \mid \overrightarrow{\mathbf{n}}}^{M}}{R_{k}}\right],
\end{aligned}
$$

where we restrict data transmitted to be non-negative. By conditioning on the independent random variable $T^{(L)}$, the approximate CDF can be expressed as Eq. (32).

\section{REFERENCES}

[1] T. Hu, E. Bigelow, J. Luo, and H. Kautz, "Tales of two cities: Using social media to understand idiosyncratic lifestyles in distinctive metropolitan areas," IEEE Transactions on Big Data, vol. 3, no. 1, pp. 55-66, Mar. 2017.

[2] F. Xia, W. Wang, T. M. Bekele, and H. Liu, "Big scholarly data: A survey," IEEE Transactions on Big Data, vol. 3, no. 1, pp. 18-35, Mar. 2017.

[3] M. M. Najafabadi, F. Villanustre, T. M. Khoshgoftaar, N. Seliya, R. Wald, and E. Muharemagic, "Deep learning applications and challenges in big data analytics," J. Big Data, vol. 2, no. 1, Mar. 2015.

[4] S. Bi, R. Zhang, Z. Ding, and S. Cui, "Wireless communications in the era of big data," IEEE Commun. Mag., vol. 53, no. 10, pp. 190-199, Oct. 2015.

[5] H. Tran, T. Duong, and H. J. Zepernick, "Delay performance of cognitive radio networks for point-to-point communications," EURASIP J. on Wireless Commun. and Networking, vol. 2012, pp. 1-14, Jan. 2012.

[6] T. M. C. Chu, H. Phan, and H. J. Zepernick, "On the performance of underlay cognitive radio networks using $\mathrm{m} / \mathrm{g} / 1 / \mathrm{k}$ queueing model," IEEE Commun. Lett., vol. 17, no. 5, pp. 876-879, May 2013.

[7] Y.-C. Lai and W.-H. Li, "A novel scheduler for proportional delay differentiation by considering packet transmission time," IEEE Commun. Lett., vol. 7, no. 4, pp. 189-191, Apr. 2003.

[8] M. Usman, H. C. Yang, and M. S. Alouini, "Extended delivery time analysis for cognitive packet transmission with application to secondary queuing analysis," IEEE Trans. Wireless Commun., vol. 14, no. 10, pp. 5300-5312, Oct. 2015.

[9] W. J. Wang, M. Usman, H. C. Yang, and M. S. Alouini, "Extended delivery time analysis for secondary packet transmission with adaptive modulation under interweave cognitive implementation," IEEE Trans. Cognit. Commun. and Netw., vol. 3, no. 2, pp. 180-189, June 2017.

[10] H. Liu, Y. Zhou, L. Tian, and J. Shi, "How can vehicular communication reduce rear-end collision probability on highway," in 2015 IEEE Global Communications Conference (GLOBECOM), Dec 2015, pp. 1-6.

[11] S. G. Cui, A. J. Goldsmith, and A. Bahai, "Energy-efficiency of mimo and cooperative mimo techniques in sensor networks," IEEE J. Sel. Areas Commun., vol. 22, no. 6, pp. 1089-1098, Aug. 2004.

[12] S. Cui, A. J. Goldsmith, and A. Bahai, "Energy-constrained modulation optimization," IEEE Trans. Wireless Commun., vol. 4, no. 5, pp. 2349 2360, Sep. 2005.

[13] R. Vaze, "Competitive ratio analysis of online algorithms to minimize packet transmission time in energy harvesting communication system," in INFOCOM, 2013 Proceedings IEEE, Apr. 2013, pp. 115-1123.

[14] X. Zheng and Z. Cai, "Real-time big data delivery in wireless networks: A case study on video delivery," IEEE Trans. Ind. Informat., vol. 13, no. 4, pp. 2048-2057, Aug. 2017. 
[15] L. Sibomana, H. J. Zepernick, H. Tran, and C. Kabiri, "Packet transmission time for cognitive radio networks considering interference from primary user," in Proc. 9th IWCMC, Jul. 2013, pp. 791-796.

[16] J. Hayes, "Adaptive feedback communications," IEEE Trans. Commun. Technol., vol. 16, no. 1, pp. 29-34, Feb. 1968

[17] A. Goldsmith, Wireless Communications. Cambridge University Press, 2005.

[18] S. Sampei and H. Harada, "System design issues and performance evaluations for adaptive modulation in new wireless access systems," Proceedings of the IEEE, vol. 95, no. 12, pp. 2456-2471, Dec. 2007.

[19] A. F. Molisch, N. B. Mehta, J. S. Yedidia, and J. Zhang, "Performance of fountain codes in collaborative relay networks," IEEE Trans. Wireless Commun., vol. 6, no. 11, pp. 4108-4119, Nov. 2007.

[20] IEEE Standard for Telecommunications and Information Exchange Between Systems - LAN/MAN Specific Requirements - Part 11: Wireless Medium Access Control (MAC) and physical layer (PHY) specifications: High Speed Physical Layer in the $5 \mathrm{GHz}$ band, IEEE Std 802.11a, Dec. 1999.

[21] A. Doufexi, S. Armour, M. Butler, A. Nix, D. Bull, J. McGeehan, and P. Karlsson, "A comparison of the hiperlan/2 and ieee 802.11a wireless lan standards," IEEE Commun. Mag., vol. 40, no. 5, pp. 172-180, May 2002.

[22] IEEE Draft Standard for Information Technology- Local and Metropolitan Area Networks- Specific Requirements- Part 15.3: Wireless Medium Access Control (MAC) and Physical Layer (PHY) Specifications for High Rate Wireless Personal Area Networks (WPAN) Amendment: HighRate Close Proximity Point-to-Point Communications, pp. 1-152, IEEE P802.15.3e/D04 2016.

[23] Unapproved Draft IEEE Standard for Local and Metropolitan Area Networks- Part 16: Air Interface for Fixed Broadband Wireless Access Systems, IEEE Std P802.16-2004/Cor1/D5 2005.

[24] A. J. Goldsmith and S.-G. Chua, "Variable-rate variable-power mqam for fading channels," IEEE Trans. Commun., vol. 45, no. 10, pp. 12181230, Oct. 1997.

[25] Q. Liu, S. Zhou, and G. B. Giannakis, "Cross-layer combining of adaptive modulation and coding with truncated arq over wireless links," IEEE Trans. Wireless Commun., vol. 3, no. 5, pp. 1746-1755, Sep. 2004.

[26] I. Adan and J. Resing, Queueing Theory. Department of Mathematics and Computing Science, Eindhoven University of Technology, Eindhoven, The Netherlands, 2001.

[27] I. S. Gradshteyn and I. M. Ryzhik, Table of Integrals, Series and Products. 7th ed. Academic Press, 2007.

[28] "Cisco visual networking index: Globalmobile data traffic forecast update 2012-2017," Tech. Rep. 1454457600805266, Cisco, Tech. Rep., 2013.

[29] "Evolved universal terrestrial radio access (E-UTRA); physical layer procedures (release 12)," Sophia-Antipolis, France, 3GGP Technical Specification 36.213 v12.0.0, Tech. Rep., Dec. 2013.

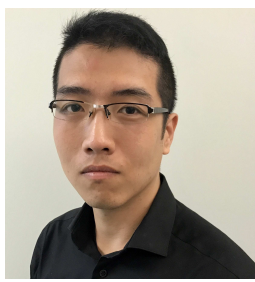

Wen-Jing Wang (S'16) is currently pursuing his $\mathrm{Ph} . \mathrm{D}$. degree in Electrical and Computer Engineering, University of Victoria, Victoria, B.C., Canada. His research interests include cognitive ratio, energy efficient communications, energy harvesting, system performance analysis and optimization.

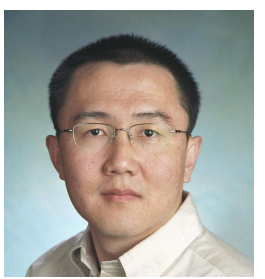

Hong-Chuan Yang (S'00, M'03, SM'07) received his Ph.D. degree in Electrical Engineering from the University of Minnesota, Minneapolis, USA, in 2003. Since September 2003, Dr. Yang has been with the Department of Electrical and Computer Engineering at the University of Victoria, Victoria, B.C., Canada, where he is now a professor. From 1995 to 1998 , Dr. Yang was a Research Associate at the Science and Technology Information Center (STIC) of Ministry of Posts \& Telecomm. (MPT), Beijing, China. His research interest include wireless channel modeling, diversity techniques, system performance evaluation, crosslayer design, and energy efficient communications.

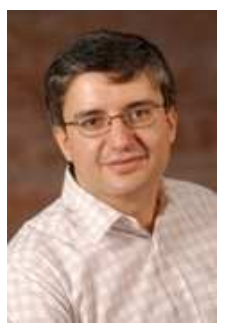

Mohamed-Slim Alouini (S'94, M'98, SM'03, F'09) was born in Tunis, Tunisia. He received the Ph.D. degree in Electrical Engineering from the California Institute of Technology (Caltech), Pasadena, CA, USA, in 1998. He served as a faculty member in the University of Minnesota, Minneapolis, MN, USA, then in the Texas A\&M University at Qatar, Education City, Doha, Qatar before joining King Abdullah University of Science and Technology (KAUST), Thuwal, Makkah Province, Saudi Arabia as a Professor of Electrical Engineering in 2009. His current research interests include the modeling, design, and performance analysis of wireless communication systems. 